\title{
Dynamics of ion sound waves in the front of the terrestrial bow shock
}

\author{
I. Giagkiozis, S. N. Walker, and M. A. Balikhin \\ Automatic Control and Systems Engineering, University of Sheffield, Sheffield, UK \\ Received: 28 February 2011 - Revised: 5 May 2011 - Accepted: 6 May 2011 - Published: 11 May 2011
}

\begin{abstract}
Single spacecraft measurements from the Cluster 3 satellite are used to identify nonlinear processes in ionsound turbulence observed within the front of the quasiperpendicular terrestrial bow shock. Ion sound waves possess spatial scales that are too small for the efficient use of multipoint measurements on inter-satellite separation scales. However, it is shown how frequency domain modelling can be applied to single spacecraft electric field data obtained using the EFW internal burst mode. The resulting characteristics of the nonlinear processes are used to argue about the possible wave sources and investigate their dynamics.
\end{abstract}

Keywords. Space plasma physics (Nonlinear phenomena; Shock waves; Wave-wave interactions)

\section{Introduction}

The ultimate aim of the analysis of plasma turbulence measurements is to characterise the processes that are responsible for transfer of energy within the plasma. The first step in this methodology is to identify the component plasma wave modes that exist within the turbulence. Once the wave spectrum has been determined it is then possible to analyse their role in the dynamical processes operating within the plasma that are responsible for the transfer of energy between different scales and also between the plasma particles and the turbulence.

The plasma wave composition can be determined using the phase differencing technique which compares the observed phase change as a wave or structure propagates between two spatial locations. This technique has been successfully applied to multi-spacecraft data in many key regions of so-

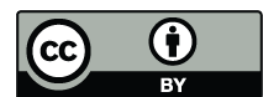

Correspondence to: S. N. Walker (simon.walker@sheffield.ac.uk) lar terrestrial plasma (Balikhin et al., 1997a,b, 1999, 2001b; Walker et al., 2004; Hobara et al., 2007a,b).

Historically 3 approaches to the identification of nonlinear processes have been used: bicoherency (Kim and Powers, 1979; Lagoutte and Hanasz, 1989), time domain modelling (Coca et al., 2001; Zhu et al., 2007) and frequency domain modelling (Ritz and Powers, 1986). The main advantage of bicoherency is that it can be applied to single point measurements. However the main weakness of this technique is that it susceptible to "historical" nonlinearities as well as nonlinearities introduced by instrumental effects such as digitisation (Walker et al., 2002). For a comprehensive review of the different methods, see Balikhin et al. (2001a); Balikhin and Walker (2005).

In order to obtain reliable results, it is important to consider the spatial separation of the observation points. The separation should be less than the coherence length of the wave packet whilst at the same time being far enough apart to allow the wave packet or structure to evolve as it propagates through the plasma. For instance, in the analysis of very low frequency mirror mode waves, wave identification is based upon the multi-point measurements available from multi-spacecraft missions such as Cluster or THEMIS whose inter-satellite separation distance is of the order of a few hundred to several thousand kilometers. However, the use of multi-spacecraft data does not permit the analysis of small scale turbulence such as ion sound waves or waves at the lower hybrid frequency. For the study of these waves, high time resolution measurements with a spatial separation of a few tens of meters are required. This can be achieved using data from Electric Fields and Waves (EFW) instrument onboard of Cluster. The ability to identify wave modes based on measurements from a single spacecraft has been demonstrated by Balikhin et al. (2005). In the current paper frequency domain modelling is used to investigate the nonlinear processes within ion sound turbulence captured by the EFW instrument operating in it's internal burst mode. The short

Published by Copernicus Publications on behalf of the European Geosciences Union. 


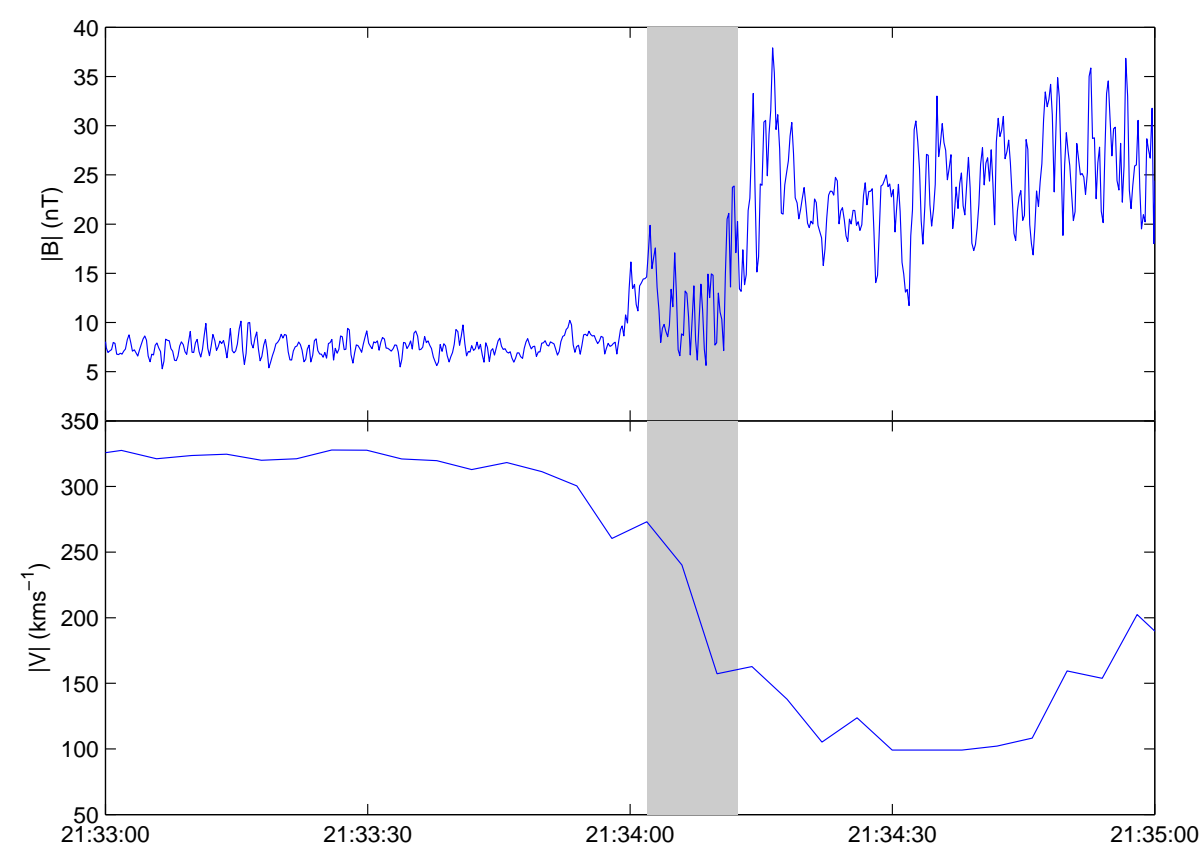

Fig. 1. The magnitude of the magnetic field (top) and the flow velocity (bottom) measured during the shock crossing on 26 February 2002.

time duration of the observed ion sound wave packets results in too few data points for the application of NARMAX based time domain analysis techniques and so frequency domain modelling techniques (Ritz and Powers, 1986; Kim and Powers, 1988; Nam and Powers, 1994) have been used.

\section{Observations}

The data used in this study were collected by the EFW (Gustafsson et al., 1997) experiment onboard the spacecraft Cluster 3. The EFW instrument measures the plasma potential using four spherical sensors situated at the ends of $44 \mathrm{~m}$ wire booms in the spin plane of the satellite. In nor$\mathrm{mal} /$ burst science modes, EFW typically computes the potential difference between opposing probes e.g. $E_{12}, E_{34}$ at rates of $25 / 450 \mathrm{~Hz}$ using a $10 / 180 \mathrm{~Hz}$ filter, respectively, as well as the individual probe potentials sampled at $5 / 10 \mathrm{~Hz}$. EFW may, when commanded, operate a triggered internal burst mode during which the instrument is capable of saving waveform snapshots of either the electric field or individual probe potentials at sampling rates of up to 36 kSamples s $^{-1}$. Timing corrections from the Cluster DWP instrument (Woolliscroft et al., 1997) were applied to improve accuracy. The EFW data obtained in this mode have been successfully used to study small scale structures within the shock front (Hobara et al., 2008). The plasma data shown in this paper were measured by the CIS HIA (Reme et al., 1997) and PEACE Johnstone et al. (1997) experiments.

On 26 February 2002 Cluster 3 was on the inbound leg of its orbit. As shown in Fig. 1 the satellite encountered the foot region of a quasi-perpendicular bow shock $\left(\theta_{\mathrm{BN}} \sim\right.$ $55^{\circ}, M_{a} \sim 4.3$ ) at around 21:34 UT. The ramp crossing occurred at around 21:34:12.5 UT. Just before the foot region is entered, Cluster 3 observed a large nonlinear structure in the magnetic field. On first inspection, this appears to be a partial crossing of the shock ramp. However, a comparison of this structure with the magnitude of the ion velocity shown in the lower panel demonstrate this not to be the case. The foot region is characterized by low frequency oscillations, typical of a quasi-perpendicular shock front. During this foot crossing the electron temperature is of the order $T_{\mathrm{e}} \sim 17 \mathrm{eV}$ and the plasma density $n_{\mathrm{i}} \sim 9.7 \mathrm{~cm}^{-3}$, resulting in a Debye length $\lambda_{\mathrm{d}} \sim 10 \mathrm{~m}$.

During this foot crossing, the EFW internal burst mode was operating and managed to capture $10 \mathrm{~s}$ waveforms of the four individual probe potentials at a sampling rate of $9 \mathrm{kHz}$ as marked by the shaded region in Fig. 1. Using this data set, it is possible to calculate two orthogonal sets of electric field measurements $E_{14}, E_{32}$ and $E_{13}, E_{42}$ for which the separation between parallel components is $\sim 62.2 \mathrm{~m}$ which corresponds to a few Debye lengths in the shock foot. The resulting simultaneous parallel electric field measurements on different sides of the satellite enable the use either phase differencing (Balikhin and Gedalin, 1993) or systems inputoutput techniques (Ritz and Powers, 1986) to compute the wave vector direction of the observed wave packets and also allow the investigation of the evolution of the wave packets as they propagate across the satellite. Since there is no electric field component normal to the spin plane, the techniques reported in this paper are limited to the analysis of the projection of the electric field onto the satellite spin plane. 
However, in most cases, the analysis can still provide sufficient information for the plasma wave mode to be identified and its linear and nonlinear interactions to be characterised.

\section{Methodology}

The availability of simultaneous measurements at two closely spaced (in comparison with the coherence length) points enable the plasma wave dynamics to be regarded as a gray box input-output system. In this situation, information about the direction of the wave propagation may be determined using the phase differencing technique. Once the wave propagation direction is determined, nonlinear interactions within the plasma may be investigated and modeled using Volterra functional series. The method employed in the present study was first described by Ritz and Powers (1986) and then further improved by Kim and Powers (1988) and Nam and Powers (1994). These authors assume that the plasma turbulence can be modeled by a second order Volterra series. This series may then be converted into the frequency domain by the use of a Fourier Transform (FT). Thus the output of the system $(Y(\xi))$ can be expressed as Eq. (1)

$$
Y(\xi)=L(\xi) X(\xi)+\frac{1}{2} \sum_{\substack{\xi_{1} \geq \xi_{2} \\ \xi=\xi_{1}+\xi_{2}}} Q\left(\xi_{1}, \xi_{2}\right) X\left(\xi_{1}\right) X\left(\xi_{2}\right)+\epsilon
$$

where $X(\xi)$ represents the FT of the input, $Y(\xi)$ the FT of the output, $L(\xi)$ the linear transfer function, and $Q\left(\xi_{1}, \xi_{2}\right)$ the quadratic transfer function. The term $\epsilon$ is the sum of errors due to truncation of the Volterra series and estimation errors. Upon expansion (1) becomes a linear equation and can thus be solved using methods such as the least squares technique or recursive least squares etc. In a further refinement McCaffrey et al. (2000) and Dudok de Witt and Krasnosel'skikh (1995) used a Continuous Wavelet Transform (CWT) for the conversion into the frequency domain as opposed to the use of the Fourier Transform. This allows much shorter data series to be analysed whilst still providing statistically robust results.

\section{Results}

\subsection{Wave packet at 21:34:01.7 UT}

Figure 2 shows the wave packet observed at around 21:34:01.7 and the results of its analysis. The upper left panel shows the input $\left(E_{13}\right.$, blue) and output ( $E_{42}$, red) waveforms used in the analysis. These waveforms display very similar structures such that the phase difference of the signals can be determined easily yet show enough differences in amplitude to conclude that the wave packet has evolved in the short time that elapsed between the two sets of parallel electric field measurements. As can be seen from the CWT spectra shown at the bottom of Fig. 2 most of the energy of the wave packet is located in the frequency range $200 \mathrm{~Hz}$ to $1000 \mathrm{~Hz}$ approximately. The magnitude of the estimated linear transfer function $(\|L(\cdot)\|)$ is shown in the left hand centre panel of Fig. 2. Values of $\|L(\cdot)\|$ greater than unity imply wave growth whilst those below imply a decay in the wave power at a particular frequency. The results suggest that there is no linear growth in the visible frequency range as the wave packet propagates. The large panel on the right shows the log of the magnitude of the quadratic transfer function $\left(\log _{10}(\|Q(\cdot, \cdot)\|)\right)$. This plot is divided into two regions. The upper region, whose lower boundary is defined by $f_{1}>0$, represents nonlinear interactions of the type $f_{1}+f_{2}=f_{3}$ i.e. the frequency of the third wave involved in this interaction is given be the summation of the frequencies of the first two waves whilst the lower portion of the plot represents nonlinear interactions of the type $f_{1}-f_{2}=f_{3}$ i.e. the frequency of the third wave is the difference between the frequencies of the first two waves. The higher the value at some particular frequency combination $f_{1}, f_{2}$ the stronger coupling between waves at $f_{1}$ and $f_{2}$ with transfer energy to the summation frequency $f_{3}$. For this particular wave packet there are several distinct features that may be identified in this plot. Firstly, in the upper triangular section that represents interactions corresponding to the summation of the two frequencies there are a number of localised maxima at frequency pairs $\left(f_{1}, f_{2}\right)=(450,450),(450,1350)$, $(450,2250) \mathrm{Hz}$. These interactions represent the generation of higher harmonics of the fundamental frequency observed within the wave packet and their effect is to move the wave energy to higher and higher frequencies. These spot frequency interactions lie within a general band of interactions that exist within both the summation and difference regions of the plot. The band in the summation interaction region is situated at about $f_{1} \approx 337.5 \mathrm{~Hz} \pm 230 \mathrm{~Hz}$ and extends along $f_{2}$ up to the Nyquist frequency with it's magnitude steadily decreasing in the direction of higher frequencies. In the difference interaction region something similar can be seen about $f_{1} \approx-450 \mathrm{~Hz} \pm 290 \mathrm{~Hz}$ which also extends along $f_{2}$ up to the Nyquist frequency, although this band is discontinuous for $f_{2} \sim 112.5 \mathrm{~Hz}$ to $\sim 562 \mathrm{~Hz}$.

A third feature of the plot, observed in the difference region, is an area of low magnitudes that extends diagonally downwards from the point $f_{1} \sim 0, f_{2} \sim 300 \mathrm{~Hz}$ (corresponding to the gap mentioned above). This feature follows the frequency relationship $f_{2}-f_{1} \approx 337 \mathrm{~Hz} \pm 225 \mathrm{~Hz}$. This combination of features in the sum and difference regions suggests that there is energy transport towards higher and higher frequencies where, according to the linear transfer function, the wave energy is dissipated to plasma due to wave damping. To check the conclusion of Balikhin et al. (2005) that ion sound turbulence was observed rather than electromagnetic whistler waves, the wave vector was estimated using the phase differencing technique and shown to be almost parallel to the direction of the wave electric field indicating electrostatic nature of the observed waves. 

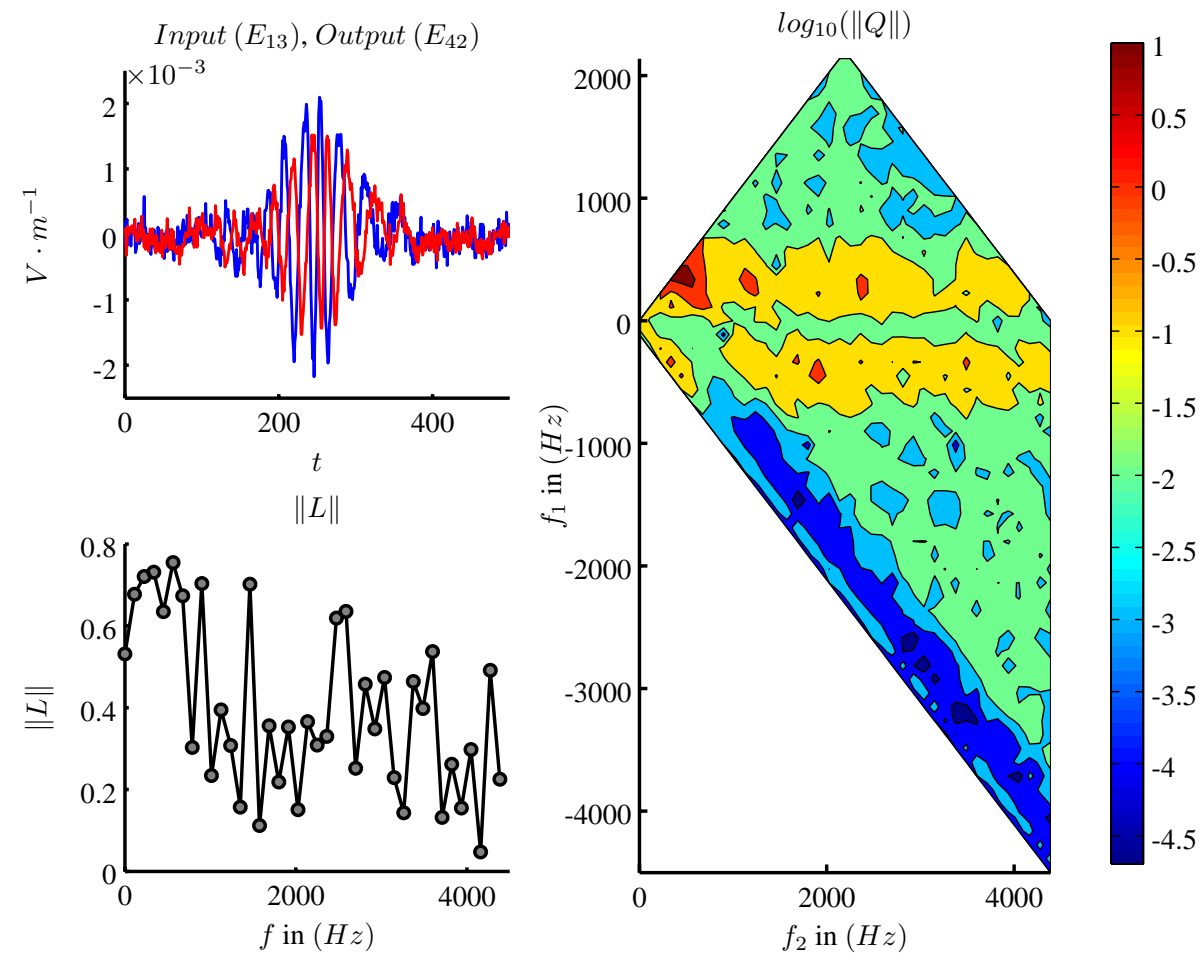

Input

Output
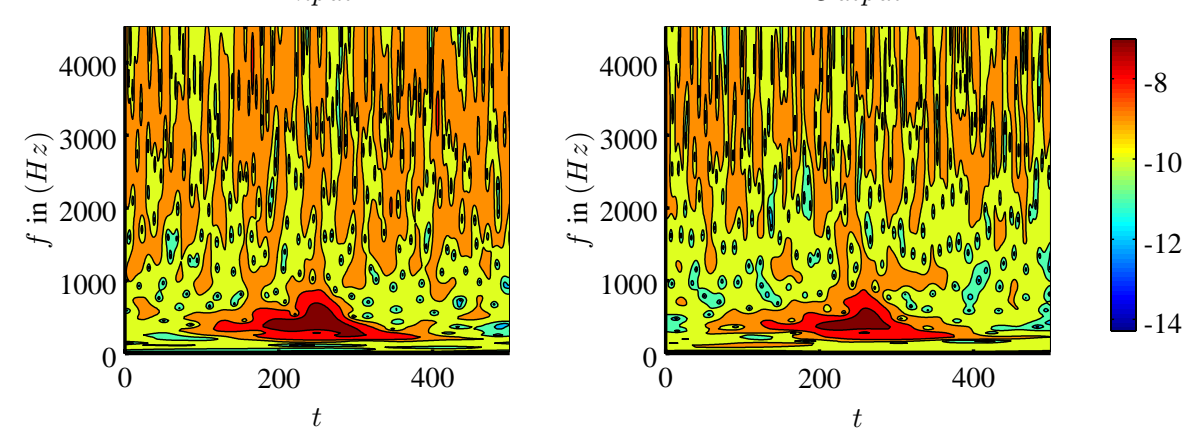

Fig. 2. Wave packet observed at 21:34:01:700 on 26 February 2002. The Input-Output electric fields (top left). The estimated linear transfer function (left middle). The estimated quadratic transfer function (top and middle right). The input ( $\left.E_{13}\right)$ CWT (bottom left) and the output $\left(E_{42}\right)$ CWT (bottom right).

\subsection{Wave packet at 21:34:02.1 UT}

The second wave packet was observed approximately $\sim 0.4 \mathrm{~s}$ after the first and is shown in Fig. 3 (which has the same format as Fig. 2). For this wave packet most of it's energy is found in the frequency range from $281.25 \mathrm{~Hz}$ to $2250 \mathrm{~Hz}$ approximately. This broader frequency span inevitably limits the range of features visible in the quadratic transfer function. In this case, as in the previous one, the estimated linear transfer function suggests that there is no linear growth, although for low frequencies it seems to have a value a little above unity. However, this is well within the statistical error of the analysis and since very little energy is located in this frequency range it could simply be due to the bias introduced by the regularization method. The quadratic transfer function again possesses very similar characteristics to those observed for the first wave packet. The two bands are simply shifted towards higher frequencies and have a wider spread. There is also evidence of the generation of higher harmonics e.g. $\left(f_{1}, f_{2}\right) \sim(1237.5,1237.5 \mathrm{~Hz})$. Analogously the band of sum interactions is located at about $f_{1} \approx 1237.5 \mathrm{~Hz} \pm 562.5 \mathrm{~Hz}$ extending along the $f_{2}$ frequency with a steadily decreasing magnitudes towards higher frequencies. The difference region is centered about $f_{1} \approx-1125 \mathrm{~Hz} \pm 618.5 \mathrm{~Hz}$ with an abrupt decrease in amplitude for $f_{2} \sim 787 \mathrm{~Hz}$ to $\sim 2250 \mathrm{~Hz}$ similar in nature with the first wave. Thus the conclusion is that the same nonlinearity is present in both wave packets since the shapes of their linear and quadratic transfer functions share a number of similar characteristics. The estimated wave vector projection for this packet is $\sim-0.04 \mathrm{~m}^{-1}$ and 

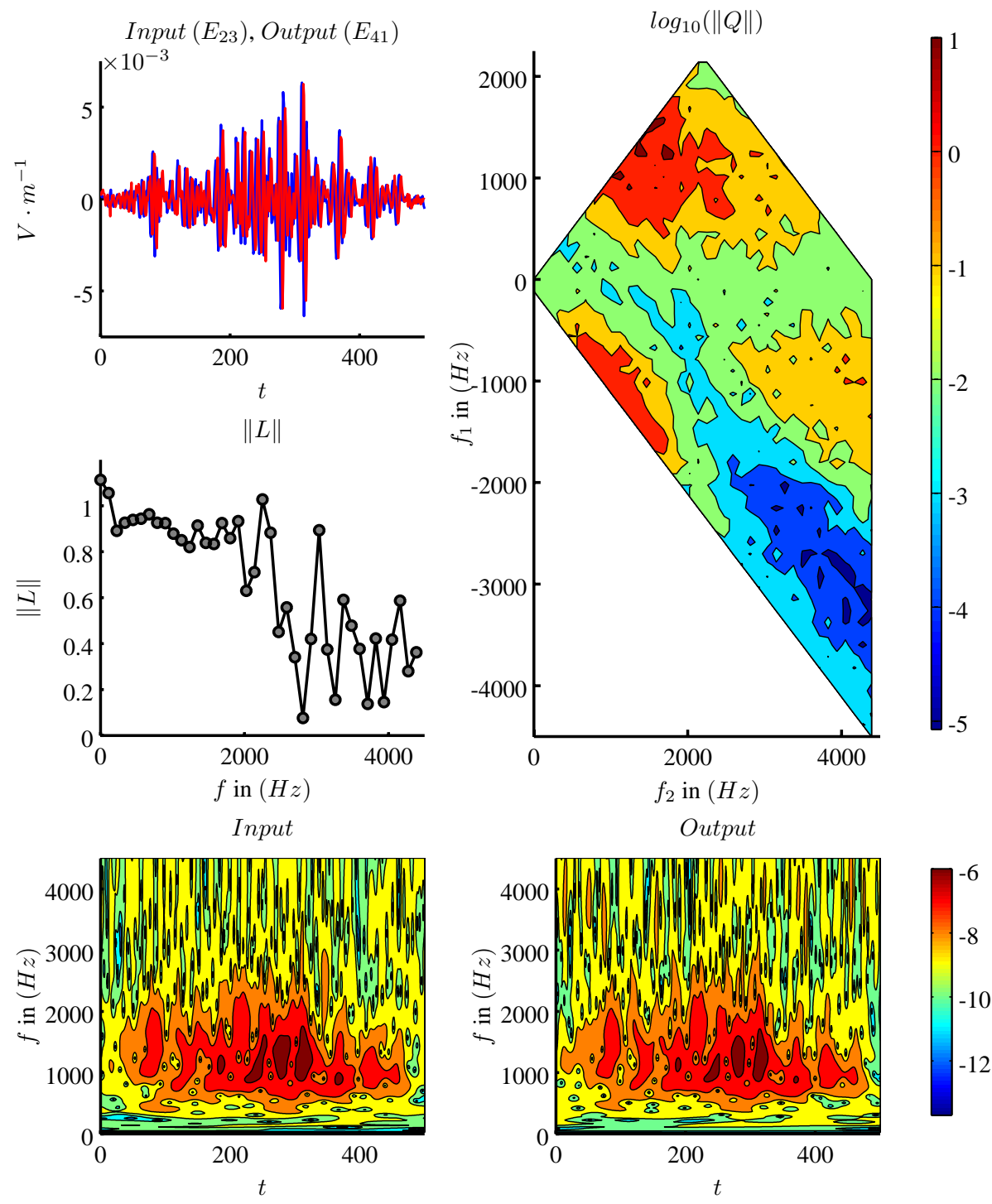

Fig. 3. Wave packet observed at 21:34:02:100 on 26 February 2002. The Input-Output electric fields (top left). The estimated linear transfer function (left middle). The estimated quadratic transfer function (top and middle right). The input $\left(E_{23}\right) \mathrm{CWT}$ (bottom left) and the output $\left(E_{41}\right)$ CWT (bottom right).

its angle with the electric field is $\sim 13.8^{\circ}$. Thus, this wave packet also appears to be propagating as an ion sound mode, similar to the first packet analysed above.

\section{Discussion and conclusions}

The main conclusion of this paper is that EFW internal burst mode data allows the application of frequency domain modelling techniques to single spacecraft data in order to identify nonlinear processes in short scale turbulence that can not be investigated using multi-satellite separation distances.

In this paper, observations of two wave packets that occurred in the foot region of a quasi-perpendicular shock were analysed using the phase differencing technique to deter- mine their propagation directions and the Ritz and Powers frequency domain modelling technique to analyse the linear and quadratic transfer functions. It was shown that both wave packets show similar characteristics, i.e. both are electrostatic ion sound mode waves. The linear transfer function for both wave packets suggests no linear growth as the wave packets propagate across the satellite. In both cases, the quadratic transfer function shows that energy is transferred to higher and higher frequencies (smaller scales).

It was thought that the main source of ion-sound turbulence within the shock front was related to plasma instabilities operating within the magnetic ramp and driven either by strong electric current or electron thermal anisotropy (Papadopoulos, 1985; Sagdeev and Galeev, 1969). However, 
observation of ion-sound waves in the foot, together with their possible propagation velocity, implies a generation mechanism operating upstream of the ramp. A comparison of wave vectors enabled (Balikhin et al., 2005) to conclude that both wave packets were generated simultaneously and the difference in the observed frequencies can be explained by a Doppler shift as they propagate in opposite directions in the plasma frame. The results of the present paper agree with these previous conclusions. They indicate that as waves propagate they simply dissipate with the transfer energy to plasma. The waves were also observed as short lived, highly coherent wave packets. Therefore they are not result of a long lived plasma instability but were more likely generated by a short lived process that operated prior to their observation. This can be related to shock non-stationarity either induced by solar wind variations or by the internal evolution of the shock front itself (Krasnosel'skikh, 1985; Krasnosel'skikh et al., 1991).

Acknowledgements. SNW was supported by a research grant from STFC.

Guest Editor M. Gedalin thanks two anonymous referees for their help in evaluating this paper.

\section{References}

Balikhin, M. A. and Gedalin, M. E.: Comparative analysis of different methods for distinguishing temporal and spatial variations, in: Proc. of START Conf., Aussois, France, vol. ESA WPP 047, pp. 183-187, 1993.

Balikhin, M. A. and Walker, S. N.: Methods of plasma turbulence analysis: Application to shock studies, in: The Physics of Collisionless Shocks, edited by: Li, G., Zank, G. P., and Russell, C. T., vol. CP781, pp. 123-128, American Institute of Physics, 2005.

Balikhin, M. A., Dudok de Wit, T., Woolliscroft, L. J. C., Walker, S. N., Alleyne, H., Krasnosel'skikh, V., Mier-Jedrzejowicz, W. A. C., and Baumjohann, W.: Experimental determination of the dispersion of waves observed upstream of a quasi-perpendicular shock, Geophys. Res. Lett., 24, 787-790, doi:10.1029/97GL00671, 1997a.

Balikhin, M. A., Walker, S. N., Dudok de Witt, T., Alleyne, H. S., Woolliscroft, L. J. C., Mier-Jedrzejowicz, W., and Baumjohann, W.: Nonstationarity and Low Frequency Turbulence at a Quasi-perpendicular Shock Front, Adv. Space Res., 20, 729-734, 1997b.

Balikhin, M. A., Alleyne, H. S. K., Walker, S. N., Treumann, R. A., Nozdrachev, M. N., and Baumjohann, W.: The role of non-linear interaction in the formation of LF whistler turbulence upstream of a quasi-perpendicular shock, J. Geophys. Res. A, 104, 1252512536, doi:10.1029/1998JA900102, 1999.

Balikhin, M. A., Bates, I., and Walker, S.: Identification of Linear and Nonlinear Processes in Space Plasma Turbulence data, Adv. Space Res., 28, 787-800, 2001a.

Balikhin, M. A., Schwartz, S., Walker, S. N., Alleyne, H. S. C. K., Dunlop, M., and Lühr, H.: Dual-spacecraft observations of standing waves in the magnetosheath, J. Geophys. Res., 106, 25395-25408, 2001b.
Balikhin, M., Walker, S., Treumann, R., Alleyne, H., Krasnoselskikh, V., Gedalin, M., Andre, M., Dunlop, M., and Fazakerley, A.: Ion sound wave packets at the quasiperpendicular shock front, Geophys. Res. Lett., 32, L24106, doi:10.1029/2005GL024660, 2005.

Coca, D., Balikhin, M. A., Billings, S. A., Alleyne, H. S. K., and Dunlop, M.: Time domain analysis of plasma turbulence observed upstream of a quasi-parallel shock, J. Geophys. Res. A, 106, 25005, doi:10.1029/2000JA000431, 2001.

Dudok de Witt, T. and Krasnosel'skikh, V.: Wavelet bicoherence analysis of strong plasma turbulence at the Earth's quasi-parallel bow shock, Phys. Plasmas, 2, 4307, 1995.

Gustafsson, G., Bostrøm, R., Holback, B., Holmgren, G., Lundgren, A., Stasiewicz, K., Åéhlen, L., Mozer, F. S., Pankow, D. Harvey, P., Berg, P., Ulrich, R., Pedersen, A., Schmidt, R., Butler, A., Fransen, A. W. C., Klinge, D., Thomsen, M., Falthammar, C.-G., Lindqvist, P.-A., Christenson, S., Holtet, J., Lybekk, B., Sten, T. A., Tanskanen, P., Lappalainen, K., and Wygant, J.: The electric field and wave experiment for the Cluster mission, Space Sci. Rev., 79, 137-156, 1997.

Hobara, Y., Walker, S. N., Balikhin, M., Pokhotelov, O. A., Dunlop, M., Nilsson, H., and Rème, H.: Characteristics of terrestrial foreshock ULF waves: Cluster observations, J. Geophys. Res. A, 112, A07202, doi:10.1029/2006JA012142, 2007a.

Hobara, Y., Walker, S. N., Dunlop, M., Balikhin, M., Pokhotelov, O. A., Nilsson, H., and Réme, H.: Mode identifcation of terrestrial ULF waves observed by Cluster: A case study, Planet. Space Sci., 55, 2237-2260, doi:10.1016/j.pss.2007.05.020, 2007b.

Hobara, Y., Walker, S. N., Balikhin, M., Pokhotelov, O. A., Gedalin, M., Krasnoselskikh, V., Hayakawa, M., André, M., Dunlop, M., Rème, H., and Fazakerley, A.: Cluster observations of electrostatic solitary waves near the Earth's bow shock, J. Geophys. Res. A, 113, A05211, doi:10.1029/2007JA012789, 2008.

Johnstone, A. D., Alsop, C., Burge, S., Carter, P. J., Coates, A. J., Coker, A. J., Fazakerley, A. N., Grande, M., Gowen, R. A., Gurgiolo, C., Hancock, B., Narheim, B., Preece, A., Sheather, P., Winningham, J. D., and Woodliffe, R. D.: Peace: A Plasma Electron and Current Experiment, Space Sci. Rev., 79, 351-398, 1997.

Kim, Y. C. and Powers, E. J.: Digital bispectral analysis and its applications to nonlinear wave interactions, IEEE Transactions on Plasma Science, 7, 120-131, doi:10.1109/TPS.1979.4317207, 1979.

Kim, K. I. and Powers, E. J.: A digital method of modeling quadratically nonlinear systems with a general random input, IEEE Transactions on Acoustics Speech and Signal Processing, 36, 1758-1769, 1988.

Krasnosel'skikh, V.: Nonlinear motions of a plasma across a magnetic field, Sov. Phys. Jetp, 62, 282-294, 1985.

Krasnosel'skikh, V. V., Balikhin, M. A., Alleyne, H. S., Klimov, S. I., Mier-Jedrzejowicz, W. A. C., Pardaens, A. K., Petrukovich, A., Southwood, D. J., Vinogradova, T., and Woolliscroft, L. J. C.: On the nature of low frequency turbulence in the foot of strong quasi-perpendicular shocks, Adv. Space Res., 11, 15-18, 1991.

Lagoutte, D. F. L. and Hanasz, J.: Application of bicoherence analysis in the study of wave interactions in space plasma, J. Geophys Res. A, 94, 435-442, 1989.

McCaffrey, D., Bates, I., Balikhin, M. A., Alleyne, H. S. C. K., Dunlop, M., and Baumjohann, W.: Experimental Method for 
Identification of Disper-Sive Three-Wave Coupling in Space Plasma, Adv. Space Res., 25, 1571-1577, doi:10.1016/S02731177(99)00670-5, 2000.

Nam, S. W. and Powers, E. J.: Application of higher order spectral analysis to cubically nonlinear system identification, IEEE Transactions on Signal Processing, 42, 1746-1765, doi:10.1109/78.298282, 1994.

Papadopoulos, K.: Microinstabilities and anomolous transport, in: Collisionless Shocks in the Heliosphere: A Tutorial Review, edited by: Stone, R. G. and Tsurutani, B. T., vol. 34 of Geophysical Monograph, pp. 59-90, American Geophysical Union, Washington, 1985.

Reme, H., Bosqued, J. M., Sauvaud, J. A., Cros, A., Dandouras, J., Aoustin, C., Bouyssou, J., Camus, T., Cuvilo, J., Martz, C., Médale, J. L., Perrier, H., Romefort, D., Rouzaud, J., D’Uston, D., Möbius, E., Crocker, K., Granoff, M., Kistler, L. M., Popecki, M., Hovestadt, D., Klecker, B., Paschmann, G., Scholer, M., Carlson, C. W., Curtis, D. W., Lin, R. P., McFadden, J. P., Formisano, V., Amata, E., Bavassano-Cattaneo, M. B., Baldetti, P., Belluci, G., Bruno, R., Chionchio, G., di Lellis, A., Shelley, E. G., Ghielmetti, A. G., Lennartsson, W., Korth, A., Rosenbauer, U., Lundin, R., Olsen, S., Parks, G. K., McCarthy, M., and Balsiger, H.: The Cluster Ion Spectrometry (CIS) Experiment, Space Sci. Rev., 79, 303-350, 1997.
Ritz, C. P. and Powers, E. J.: Estimation of nonlinear transfer functions for fully developed turbulence, Physica Scripta, 20D, 320, 1986.

Sagdeev, R. Z. and Galeev, A. A.: Nonlinear Plasma Theory, Benjamin, White Plains, N.Y., 1969.

Walker, S. N., Balikhin, M. A., Bates, I., and Huff, R.: An Investigation into Instrumental Nonlinear Effects, Adv. Space Res., 30, 2815-2820, 2002.

Walker, S. N., Sahraoui, F., Balikhin, M. A., Belmont, G., Pinon, J. L., Rezeau, L., Alleyne, H., Cornilleau-Wehrlin, N., and André, M.: A comparison of wave mode identification techniques, Ann. Geophys., 22, 3021-3032, doi:10.5194/angeo-223021-2004, 2004.

Woolliscroft, L. J. C., Alleyne, H. S. C., Dunford, C. M., Sumner, A., Thompson, J. A., Walker, S. N., Yearby, K. H., Buckley, A., Chapman, S., and Gough, M. P.: The Digital Wave-Processing Experiment on Cluster, Space Sci. Rev., 79, 209-231, 1997.

Zhu, D., Billings, S. A., Balikhin, M. A., Wing, S., and Alleyne, H.: Multi-input data derived Dst model, J. Geophys. Res. A, 112, A06205, doi:10.1029/2006JA012079, 2007. 